UDC 004.822

\title{
QUERY ANSWERING OVER FACT BASES IN ZADEH LOGIC
}

\author{
(This work is partially supported by RFBR grant № 12-01-00705)
}

Plesniewicz G.S., Ph.D. (Tech.Sc.), professor

(National Research University “MPEI”, Krasnokazarmennaya St., 14, Moscow, 111250, Russian Federation, salve777@mail.ru)

Аннотация. Let $\mathbf{L}$ be Zadeh logic i.e. the fuzzy propositional logic based on triangular norm $\min (x, y)$. A fact in $\mathbf{L}$ is an expression of the form $r \leq \varphi \leq s$ where $\varphi \in \mathbf{L}$ and $0 \leq r \leq s \leq 1$. In a fuzzy interpretation I of $\mathbf{L}$ every fact is true or false, and $\mathrm{I}(r \leq \varphi \leq s)=1$ if and only if the two-side inequality $r \leq \mathrm{I}(\varphi) \leq s$ is satisfied. Thus, the set $\mathbf{F L}$ of all facts in $\mathbf{L}$ defines a crisp logic with fuzzy interpretations. Logical consequence "|=" in the logic $\mathbf{F L}$ is defined as usual: for any set $E$ of facts and any fact $\alpha, E \mid=\alpha$ if there are no an interpretation I such that $\mathrm{I}(\alpha)=$ 1 and $\mathrm{I}(\beta)=1$ for all $\beta \in E$.. But in the logic FL there is also strong logical consequence $\left|=^{*}: E\right|=^{*} r \leq \varphi \leq s$ if $E$ $\mid=r \leq \varphi \leq s$ and it is not true that $E \mid=r^{\prime} \leq \varphi \leq s$ with $r^{\prime}>r$ and not true $E \mid=r \leq \varphi \leq s^{\prime}$ with $s^{\prime}<s$.

A fact base is a finite set $F$ of facts: $F=\left\{r_{\mathrm{i}} \leq \varphi_{\mathrm{i}} \leq s_{\mathrm{i}} \mid 1 \leq i \leq n\right\}$. One can consider the set $K=\left\{\varphi_{\mathrm{i}} \mid 1 \leq i \leq n\right\}$ as a fuzzy knowledge base and $F$ as an instance of $K$. A query is an expression of the form ? $\psi$ where $\psi \in \mathbf{L}$. The answer to the query to the fact base $F$ is the fact $r \leq \psi \leq s$ such that $F \mid=* r \leq \psi \leq s$.

The problem of query answering over fact bases in FL can be solved by analytical tableaux method. The method results in an algorithm with the exponential worst-case estimate (relatively to the size of $F \cup\{\kappa\}$ where $\kappa$ is a query). However, consider the situation when the knowledge base $K$ and the query $\kappa$ are fixed but fact bases $F$ are arbitrary instances of $K$. Then, it is possible to answer query $\kappa$ to fact bases $F$ quickly. But preliminary we should deal with the parametric fact base associated with $K$.

Under a parametric fact we mean an expression of the form $a \leq \varphi \leq b$ where $a$ and $b$ are not numbers but parameters - variables with values in [0,1]. The parametric fact base associated with $K$ is $P=\left\{a_{\mathrm{i}} \leq \varphi_{\mathrm{i}} \leq b_{\mathrm{i}} \mid 1 \leq i \leq n\right\}$ where $a_{\mathrm{i}}, b_{\mathrm{i}}$ are different parameters. Thus, if we replace the parameters by specific numbers from [0,1] (with adherence to corresponding inequality) we obtain a specific fact base which is an instance of the parametric fact base $P$.

One can also consider query answering over parametric fact bases. Let $P$ be a parametric fact base and ? $\psi$ be a query. The answer to the query is the expression $g \leq \psi \leq h$ such that $K \lambda \mid=* g \lambda \leq \psi \leq h \lambda$ for any substitution $\lambda$ of numbers from $[0,1]$ for the parameters from $K$. Here $g$ and $h$ are appropriate expressions with the parameters from $K$.

Using the analytical tableaux method, we show how to design an algorithm for finding the expressions $g$ and $h$ for a given knowledge base $K$. So, let a knowledge base $K=\left\{\varphi_{\mathrm{i}} \mid 1 \leq i \leq n\right\}$ and a query $\kappa$ : ? $\psi$ be fixed. Suppose we need to answer the query to any fact base $F=\left\{r_{\mathrm{i}} \leq \varphi_{\mathrm{i}} \leq s_{\mathrm{i}} \mid 1 \leq i \leq n\right\}$. Then we (1) apply the algorithm to the parametric fact base $P=\left\{a_{\mathrm{i}} \leq \varphi_{\mathrm{i}} \leq b_{\mathrm{i}} \mid 1 \leq i \leq n\right\}$ and obtain the expressions $g$ and $h$; (2) apply the substitution $\lambda=\left\{a_{\mathrm{i}} / r_{\mathrm{i}}, b_{\mathrm{i}} / s_{\mathrm{i}} \mid 1 \leq i \leq n\right\}$ to $g$ and $h$; thus, we obtain the answer $g \lambda \leq \psi \leq h \lambda$.

Ключевые слова: query, fact base, Zadeh logic, knowledge base, contrary condition, inequalities.

\section{Introduction. Main definitions}

Let $\mathbf{L}$ be Zadeh logic i.e. the propositional fuzzy logic based on a triangular norm $\min \{x, y\}$. The syntax of $\mathbf{L}$ is the same as the syntax of usual propositional logic. The semantics of $\mathbf{L}$ is defined by interpretations $\mathrm{I}: \mathbf{L} \rightarrow$ $[0,1]$ satisfying the following conditions for any formulas $\varphi, \psi \in \mathbf{L}$ :

$\mathrm{I}(\neg \varphi)=1-\mathrm{I}(\varphi), \mathrm{I}(\varphi \wedge \psi)=\min \{\mathrm{I}(\varphi), \mathrm{I}(\psi)\}$,

$\mathrm{I}(\varphi \vee \psi)=\max \{1-\mathrm{I}(\varphi), 1-\mathrm{I}(\psi)\}$

$\mathrm{I}(\varphi \rightarrow \psi)=\max \{1-\mathrm{I}(\varphi), \mathrm{I}(\psi)\}$.

We associate with each formula $\varphi \in \mathrm{L}$ and numbers $r, s(0 \leq r \leq s \leq 1)$ the sentence (fact) $r \leq \varphi \leq s$ which is true or false in $\mathrm{I}$, and $\mathrm{I}(r \leq \varphi \leq s)=1 \Leftrightarrow \Leftrightarrow_{\mathrm{df}} r \leq \mathrm{I}(\varphi) \leq s$. (We also write $\varphi \geq r$ instead of $r \leq \varphi \leq 1$ and $\varphi \leq r$ instead of $0 \leq \varphi \leq r$.) A fact base $F$ is a finite set of facts.

Let FL denote the set of all facts for L. Thus, FL is a crisp logic with fuzzy interpretations. As in any logic, there is the logical consequence relation $\mid=$ in $\mathbf{F L}$ : for any $E \subseteq \mathbf{F L}$ and $\alpha \in \mathbf{F L}$

$E \mid=\alpha \Leftrightarrow{ }_{\text {df }}$ there is no interpretation I such that $\mathrm{I}(\alpha)=0$ and $\mathrm{I}(\beta)=1$ for all $\beta \in E$.

But in FL there is also strong logical consequence $\mid=*$ :

$E\left|{ }^{*} r \leq \varphi \leq s \Leftrightarrow{ }_{\mathrm{df}} E\right|=r \leq \varphi \leq s$ and it is not true that $E \mid=r^{\prime} \leq \varphi \leq s$ with $r^{\prime}>r$ and $E \mid=r \leq \varphi \leq s^{\prime}$ with $r^{\prime}>r$. 
A knowledge base is a finite set $K$ of formulas from $\mathbf{L}: K=\left\{\varphi_{\mathrm{i}} \mid 1 \leq i \leq n\right\}$. Let us choose numbers $0 \leq r_{\mathrm{i}} \leq s_{\mathrm{i}} \leq$ $1(1 \leq i \leq n)$; then the fact base $F=\left\{r_{\mathrm{i}} \leq \varphi_{\mathrm{i}} \leq s_{\mathrm{i}} \mid 1 \leq i \leq n\right\}$ is an instance of the knowledge base $K$. An expression of the form ? $\psi$ is a query to the fact bases - instances of $K$ if $\psi$ is a formula of $\mathbf{L}$ in the signature of $K$. The $a n$ swer to a query ? $\psi$ to a fact base $F$ is the fact $r \leq \psi \leq s$ such that $F \mid=* r \leq \psi \leq t$.

Example 1. Let us consider the knowledge base $K=\{p \wedge q, q \rightarrow r\}$ and its instance (the fact base) $F=\{0.7 \leq$ $p \wedge q, 0.4 \leq q \rightarrow r \leq 0.6\}$. Let $\kappa=? p \wedge \neg r$ be the query to $F$. Then $0.4 \leq p \wedge \neg r \leq 0.6$ is the answer to $\kappa$.

Under a parametric fact we mean an expression of the form $a \leq \varphi \leq b$ where $a$ and $b$ are not numbers but parameters - variables with values in $[0,1]$. A parametric fact base $P$ for a knowledge base $K=\left\{\varphi_{\mathrm{i}} \mid 1 \leq i \leq n\right\}$ is the set of parametric facts with different parameters: $P=\left\{a_{\mathrm{i}} \leq \varphi_{\mathrm{i}} \leq b_{\mathrm{i}} \mid 1 \leq i \leq n\right\}\left(a_{\mathrm{i}} \neq b_{\mathrm{j}}\right.$ if $\left.i \neq j\right)$.

Let $\lambda$ be a substitution numbers for parameters: $\lambda=\left\{r_{\mathrm{i}} / a_{\mathrm{i}}, s_{\mathrm{i}} / b_{\mathrm{i}} \mid 1 \leq i \leq n\right\}\left(r_{\mathrm{i}} \leq s_{\mathrm{i}}\right)$. Then, applying $\lambda$ to $P$ we obtain the fact base $P \lambda=\left\{r_{\mathrm{i}} \leq \varphi_{\mathrm{i}} \leq s_{\mathrm{i}} \mid 1 \leq i \leq n\right\}$. One can put a query ? $\psi$ to the parametric fact base $P$ for a knowledge base $K$. Then the answer to this query is the expression $g \leq \psi \leq h$ such that $K \lambda \mid=^{*} g \lambda \leq \psi \leq h \lambda$ for any substitution $\lambda$ where $g$ and $h$ are some expressions containing parameters from $K$.

Example 2. Let the knowledge base $K$ and the query $\kappa$ be the same as in Example 1. The parametric fact base for the knowledge base is $P=\{a \leq p \wedge q \leq b, c \leq q \rightarrow r \leq d\}$. Then $g \leq p \wedge \neg r \leq h$ is the answer to $\kappa$ where

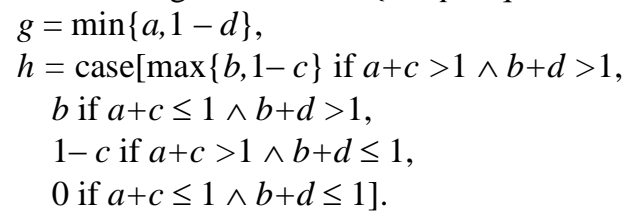

\section{Query answering to fact bases}

Let $\mathbf{M}$ be the set of all sentences from FL of the forms $\alpha \leq c, \alpha<c, \alpha \geq c$ and $\alpha>c$. Obviously, the problem of logical consequence for logic $\mathbf{F L}$ is reduced to the problem of inconsistency for logic $\mathbf{M}$ since

$F \mid=r \leq \alpha \leq s \Leftrightarrow F^{+} \cup\{\alpha>r\}$ and $F^{+} \cup(r<s)$ are inconsistent sets

where $F^{+}=\{\beta \leq s, \beta \geq r \mid(r \leq \beta \leq s) \in F\}$.

The method of analytical tableaux can be applied to solve the problem of inconsistency in $\mathbf{M}$ [2]. In Table 1 there are the inference rules for the logic $\mathrm{M}$. This method also can be applied to the problem of finding answers to queries to fact bases. We show by example, how to do it.

Table 1

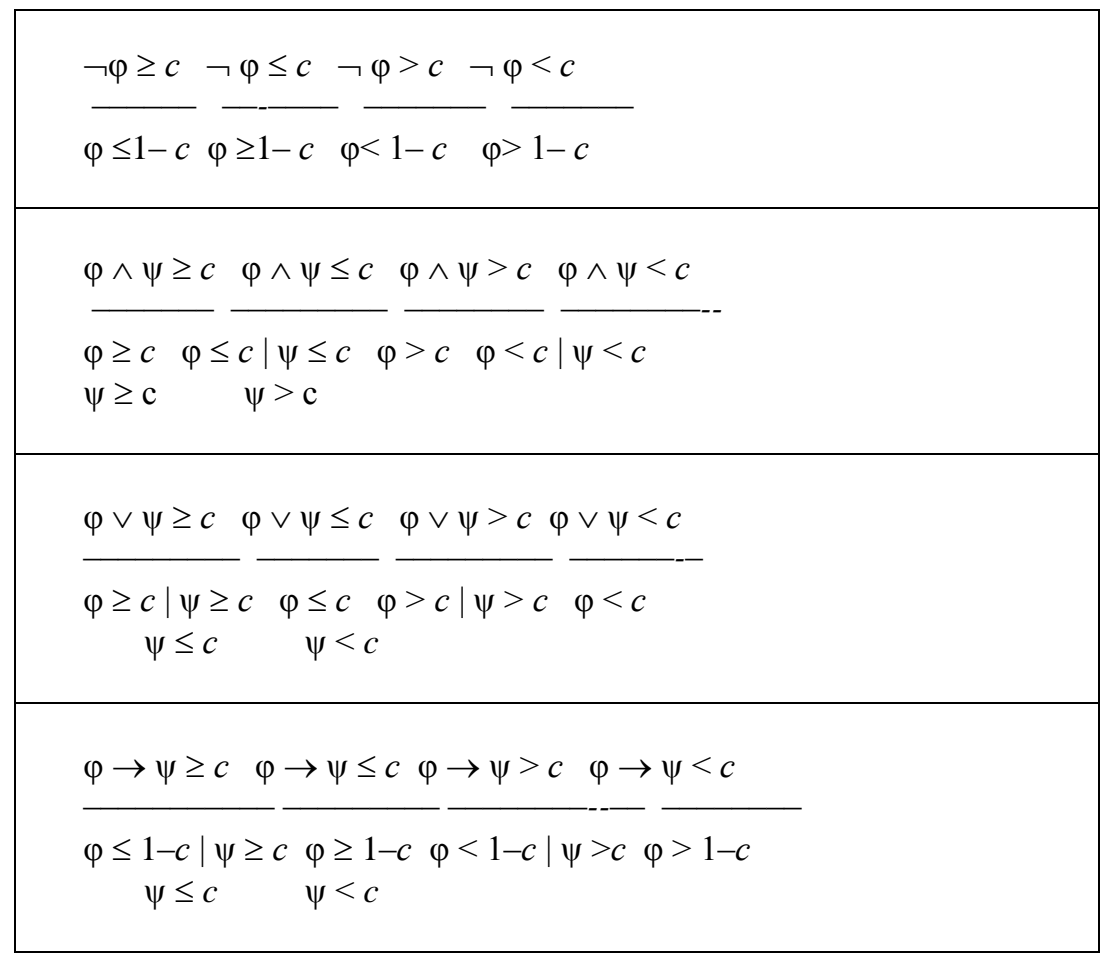

Example 3. Let the knowledge base $K$, the fact base $F$ and the query $\kappa$ be such as in Example 1 . The fact base $F$ can be replaced with the equivalent fact base $F^{+}=\{p \wedge q \geq 0.7, q \rightarrow r \geq 0.4, q \rightarrow r \leq 0.6\}$. Then $F^{+} \mid=x \leq p \wedge \neg$ $r \leq y$ if and only if the following two sets are inconsistent: 
$\mathrm{F}_{1}=\{p \wedge q \geq 0.7, q \rightarrow r \geq 0.4, q \rightarrow r \leq 0.6, p \wedge \neg r \leq x\}$

$\mathrm{F}_{2}=\{p \wedge q \geq 0.7, q \rightarrow r \geq 0.4, q \rightarrow r \leq 0.6, \mathrm{p} \wedge \neg \mathrm{r}>y\}$.

In Figure 1 the deduction trees $A$ and $B$ for these sets are shown. In A the branch (1) is closed since it contains contrary inequalities $q \geq 0.7$ and $q \leq 0.6$. The branch (2) will be closed if we choose $y$ such that inequalities $r<1-y$ and $r \geq 0.4$ becomes contrary. Also (2) will be closed if $y=1$ (then $p>1$ and that is impossible). Hence, (2) is closed if and only if $y=1$ or $0.4 \geq 1-y$, i.e. $y \geq 0.6$. Therefore, $h=\min y=0.6$. In B branches (1) and (2) are closed since they contain contrary inequalities $q \geq 0.7$ and $q \leq 0.6$. Clearly, branch (3) is closed if and only if $1-x$ $=1$, i.e. $x=0$. Therefore, $g=0$. Hence, $p \wedge \neg r \leq 0.6$ is the answer to $\kappa$.

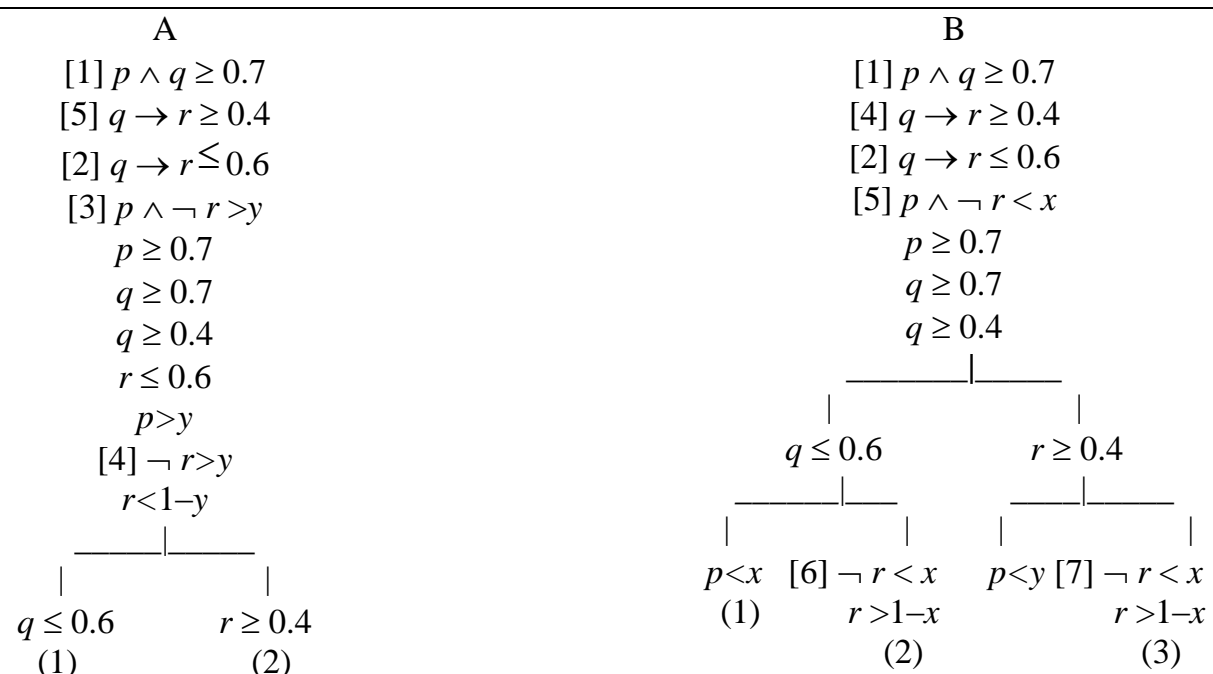

Figure 1. Deduction trees

Remark. The similar method has been offered in [1].

\section{Query answering for parametric fact bases}

One can find answers to queries to parametric fact bases by applying analytical tableaux method. Here is an example (how to do it).

Example 4. Let the knowledge base $F$, the query $\kappa$ and the parametric fact base $P$ are such as in Example 2. In Figure 2 and Figure 3 the deduction trees for the sets $P_{1}=P \cup\{p \wedge \neg r>y\}$ and $P_{2}=P \cup\{p \wedge \neg r<x\}$ are shown.

In the first tree, consider two inequalities $q \geq a$ and $q \leq 1-c$ which lie on branch (1). Clearly, they are contrary (inconsistent) if and only if $a>1-c$ i.e. $a+c>1$. We say that $(q \geq a, q \leq 1-c)$ is a candidate contrary pair and $a+c>1$ is a condition of its contrariness.

In Table 2 there are all the candidate contrary pairs together with the contrariness conditions and with references to the branches closed by the contrary pairs. From the table we see that pairs 1 and 4 (and also pairs 2 and 3 ) block up all branches of the tree. Therefore, the tree in Figure 2 is closed if and only if the following condition is satisfied:

$(a+c>1 \wedge y \geq 1-c) \vee(b+d>1 \wedge y \geq b)$

Table 2

\begin{tabular}{|llll|}
\hline 1 & $(q \leq 1-c, q \geq a)$ & $a+c>1$ & $(1),(3)$ \\
2 & $(q \leq b, q \geq 1-d)$ & $b+d>1$ & $(3),(4)$ \\
3 & $(p \leq b, p>y)$ & $y \geq b$ & $(1),(2)$ \\
4 & $(q \leq 1-c, q \geq a)$ & $y \geq 1-c$ & $(2),(4)$ \\
\hline
\end{tabular}

So, $h=\min \{y \mid y$ satisfies (1) $\}$. Depending on what conditions $a+c>1$ and $b+d>1$ are true or false the condition (2.1) is reduced to:

$$
\begin{array}{ll}
y \geq 1-c \vee y \geq b & \text { if } a+c>1 \text { and } b+d>1, \\
y \geq b & \text { if } a+c \leq 1 \text { and } b+d>1,
\end{array}
$$




\begin{tabular}{ll}
\hline & \\
0 & if $a+c>1$ and $b+d \leq 1$,
\end{tabular}

From this we obtain

$h=\operatorname{case}[\max \{b, 1-c\}$ if $a+c>1 \wedge b+d>1$,

$b$ if $a+c \leq 1 \wedge b+d>1$,

$1-c$ if $a+c>1$ and $b+d \leq 1$,

0 if $a+c \leq 1$ and $b+d \leq 1]$.

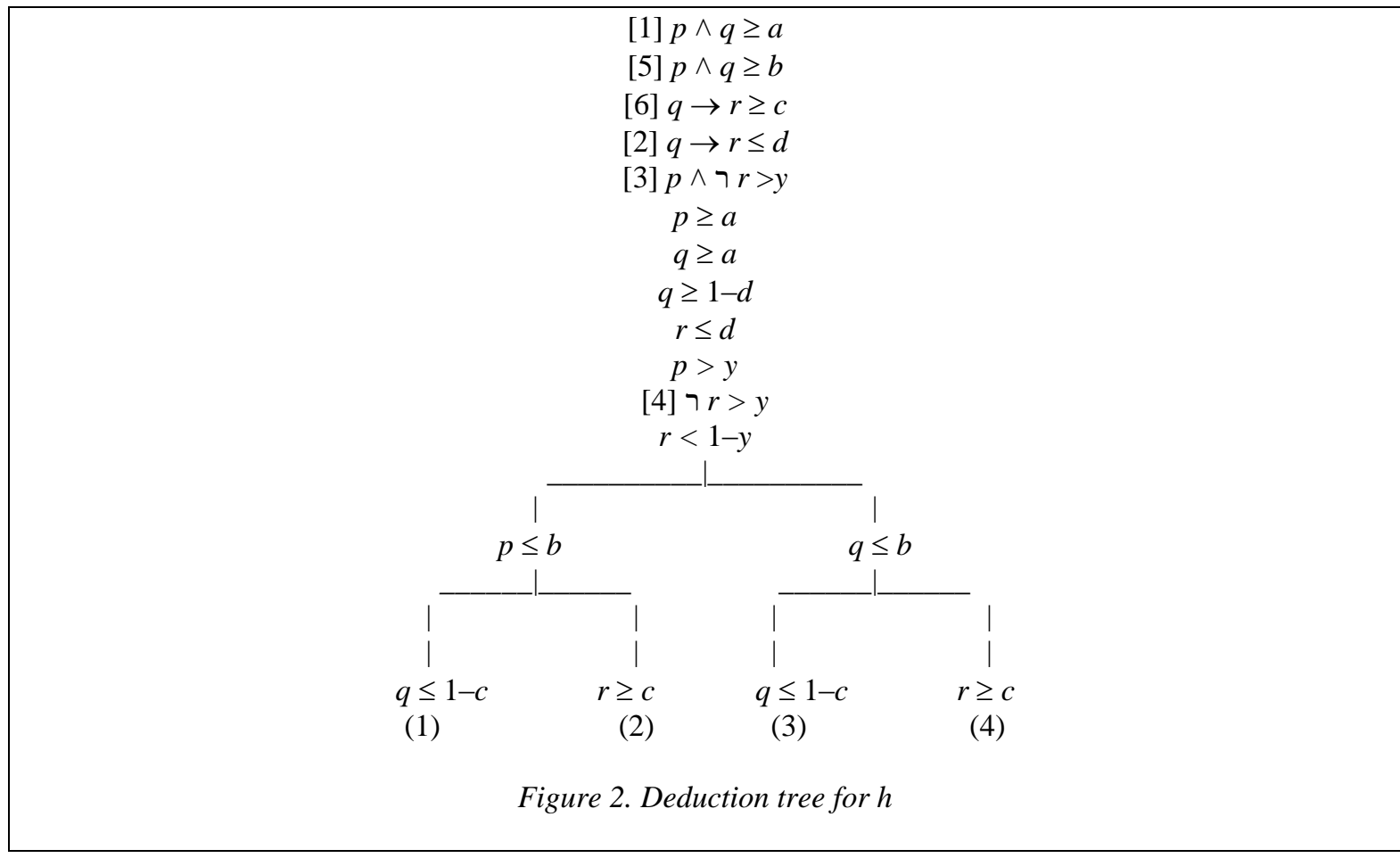

In a similar way, considering the tree in Figure 3, we find: $g=\min \{a, 1-d\}$.

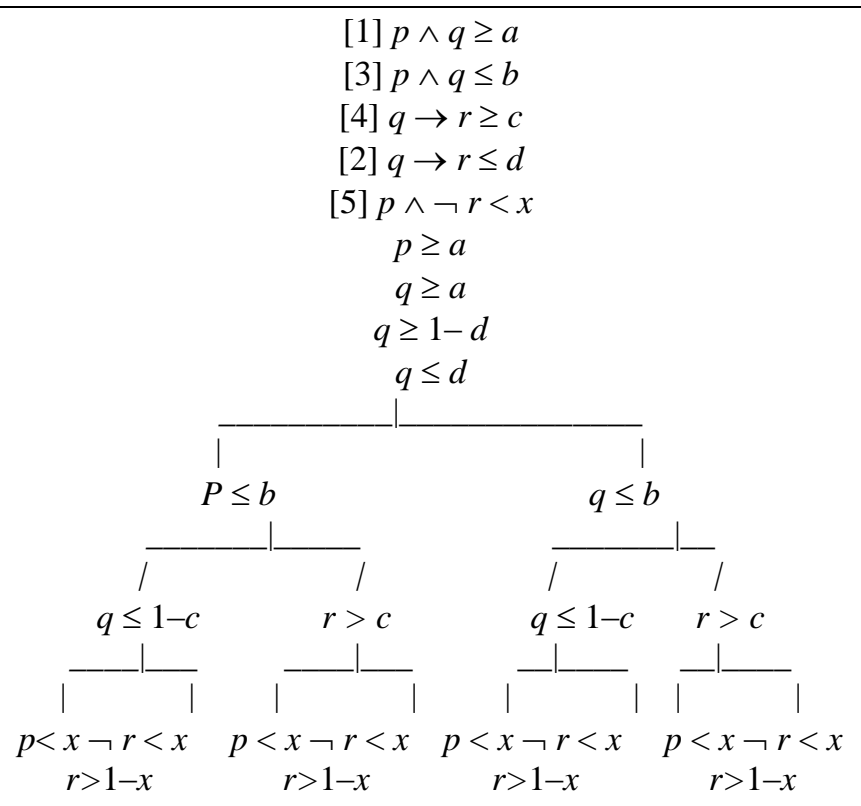

Figure 3. Deduction tree for $g$ 
Example 5. Let the knowledge base $K$, the parametric fact base $P$, the query $\kappa$, and the expressions $g$ and $h$ be such as in Example 4. Let us take the substitution $\lambda=\{a / 0.7, b / 1, c / 0.4, d / 0.6\}$. Applying $\lambda$ to $P, g$ and $h$, we obtain

$P \lambda=F=\{0.7 \leq p \wedge q, 0.4 \leq q \rightarrow r \leq 0.6\}, g \lambda=0.4, g \lambda=0.4$.

Thus, we have the answer $0.4 \leq p \wedge \neg r \leq 0.6$ to the query $p \wedge \neg r$ to the fact base $F$.

Let us consider a general situation when a knowledge base $K$, a query $\kappa$ and a parametric fact base $P$ for $K$ are arbitrary. Let $P$ have parameters $a_{\mathrm{i}}(i=1,2, \ldots)$. Suppose we construct the deduction trees $\mathrm{T}_{1}$ and $\mathrm{T}_{2}$ for the sets $P \cup\{\kappa<x\}$ and $P \cup\{\kappa>y\}$. It is easy to see that in $\mathrm{T}_{1}$ there can be candidate pairs and contrary conditions of the following forms:

$$
\begin{array}{ll}
\left(p \geq a_{\mathrm{j}}, p \leq a_{\mathrm{k}}\right), & a_{\mathrm{j}}>a_{\mathrm{k}}, \\
\left(p \geq 1-a_{\mathrm{j}}, p \leq a_{\mathrm{k}}\right), & a_{\mathrm{j}}+a_{\mathrm{k}}<1, \\
\left(p \geq a_{\mathrm{j}}, p \leq 1-a_{\mathrm{k}}\right), & a_{\mathrm{j}}+a_{\mathrm{k}}>1, \\
\left(p \geq a_{\mathrm{j}}, p<x\right), & x \leq a_{\mathrm{j}}, \\
\left(p \geq 1-a_{\mathrm{j}}, p<x\right), & x \leq 1-a_{\mathrm{j}}, \\
\left(p>1-x, p \leq a_{\mathrm{j}}\right), & x \leq 1-a_{\mathrm{j}}, \\
\left(p>1-x, p \leq 1-a_{\mathrm{j}}\right), & x \leq a_{\mathrm{j}}, \\
(p>1-x, p>x), & x \leq 1 / 2 .
\end{array}
$$

In the first tree, consider two inequalities $q \geq a$ and $q \leq 1-c$ which lie on branch (1). Clearly, they are contrary (inconsistent) if and only if $a>1-c$ i.e.

Every candidate pair in $\mathrm{T}_{1}$ blocks some branches. Let $\mathrm{b}(\pi)$ denote the set of branches which is blocked up by pair $\pi$, and let $\mathrm{c}(\pi)$ denote the contrary condition for pair $\pi$. Also, let $\mathrm{b}(S)=\mathrm{U}\{\mathrm{b}(\pi) \mid \pi \in E\}$ and $\mathrm{c}(S)=\Lambda\{\mathrm{c}(\pi) \mid$ $\pi \in S\}$ where $S$ is a set of candidate pairs.

A set $S$ of candidate pairs is a covering if $\mathrm{b}(S)$ coincides with the set of all branches of $\mathrm{T}_{1}$. Thus, if $S$ is a covering and the condition $\mathrm{c}(S)$ is satisfied with a given substation $\sigma$ then the tree $\mathrm{T}_{1} \sigma$ is closed. A covering $S$ is (locally) minimal if $S \backslash\{\pi\}$ is not a covering for each $\pi \in S$.

Let $S_{1}, S_{2}, \ldots, S_{\mathrm{m}}$ be all minimal coverings for $\mathrm{T}_{1}$. Take the condition $\mathrm{C}=\mathrm{c}\left(S_{1}\right) \vee \mathrm{c}\left(S_{2}\right) \vee \ldots \vee \mathrm{c}\left(S_{\mathrm{m}}\right)$. Thus, C is a disjunction of conjunctions made of inequalities of the form: $a_{\mathrm{j}}>a_{\mathrm{k}}, a_{\mathrm{j}}+a_{\mathrm{k}}<1, a_{\mathrm{j}}+a_{\mathrm{k}}>1, x \leq a_{\mathrm{j}}, x \leq 1-a_{\mathrm{j}}, x$ $\leq 1 / 2$.

Let $R$ be the set of all conditions which are occurred in $\mathrm{C}$ and have no the variable $x$. Let $\theta$ be any assignment of truth values 0 or 1 to the conditions from $R$, i.e. $\theta: R \rightarrow\{0,1\}$. One can consider $\theta$ as a substitution truth values for inequalities. Thus, $\mathrm{C} \theta$ has the form $\mathrm{C}_{1}^{\theta} \vee \mathrm{C}_{2}{ }_{2} \vee \ldots \vee \mathrm{C}_{\mathrm{m}}{ }_{\mathrm{m}}$ where $\mathrm{C}^{\theta}{ }_{\mathrm{i}}=\mathrm{c}\left(S_{\mathrm{i}}\right) \theta$ and $\mathrm{C}_{\mathrm{i}}^{\theta}$ has the form $(x \leq$ $\left.e_{\mathrm{i} 1}\right) \wedge\left(x \leq e_{\mathrm{i} 2}\right) \wedge \ldots \wedge\left(x \leq e_{\mathrm{iq}(\mathrm{i})}\right)$. Let us denote $\mathrm{r}\left(\mathrm{C}^{\theta}{ }_{\mathrm{i}}\right)=\left\{e_{\mathrm{i} 1}, e_{\mathrm{i} 2}, \ldots, e_{\mathrm{iq}(\mathrm{i}\}}\right\}$.

It is clear that:

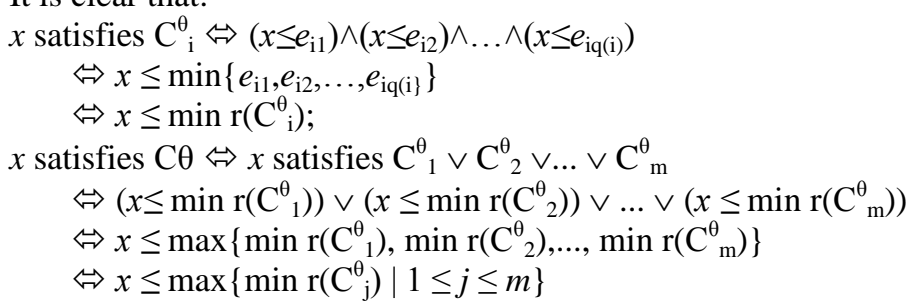

Let $\theta^{*}$ be the conjunction of the inequalities from $R$ or their negations. We include in $\theta^{*}$ an inequality if $\theta$ assigns 1 , and the contrary inequality if $\theta^{*}$ assigns 0 , i.e.

$\theta^{\#}=(\Lambda\{\sigma \mid \sigma \in R, \theta(\sigma)=1\}) \wedge(\Lambda\{\neg \sigma \mid \sigma \in \mathrm{K}, \theta(\sigma)=0\})$.

We have

$\mathrm{C}=\Lambda\left\{\theta^{\#} \rightarrow \mathrm{C} \theta \mid \theta: R \rightarrow[0,1]\right\}$

This can be understood considering the following example.

Example 6. Let $\alpha$ be a formula of propositional variables $p, q$ and $r: \alpha=\alpha[p, q, r]$. Then

$\alpha[p, q, r]=(p \wedge q \rightarrow \alpha[1,1, r]) \wedge(p \wedge \neg q \rightarrow \alpha[1,0, r]) \wedge$ $(\neg p \wedge q \rightarrow \alpha[0,1, r]) \wedge(\neg p \wedge \neg q \rightarrow \alpha[0,0, r])$.

Indeed, for example, if $p=0, q=1$ then in the right part of this equality we have $\alpha[0,1, r]$. Hence, the equality is true for $p=0, q=1$.

From (2) and (3) we obtain

$g=\max \{x \mid x$ satsfies $C\}$

$=\operatorname{case}\left[\max \left\{\min r\left(\mathrm{C}_{\mathrm{j}}^{\theta}\right) \mid 1 \leq j \leq m\right)\right\}$ if $\left.\left.\theta^{\#} \mid \theta: R \rightarrow[0,1]\right\}\right]$.

In the similar way we obtain the expression for $h$ (see Table 3 ).

Table 3

$g=\operatorname{case}\left[\max \left\{\min r\left(\mathrm{C}_{\mathrm{j}}^{\theta}\right) \mid 1 \leq j \leq m\right\}\right.$ if $\left.\left.\theta^{\#} \mid \theta: R \rightarrow[0,1]\right\}\right]$ 
$h=\operatorname{case}\left[\max \left\{\min \mathrm{r}\left(\mathrm{C}_{\mathrm{j}}^{\theta}\right) \mid 1 \leq j \leq m\right\}\right.$ if $\left.\left.\theta^{\#} \mid \theta: R \rightarrow[0,1]\right\}\right]$

Example 7. In the tree $\mathrm{T}_{2}$ (Figure 3 ) there are the candidate pairs which are written in Table 3 . From here $\mathrm{R}=\{a+c>1, b+d>1\}$. There are exactly four substitutions

$$
\begin{aligned}
& \theta_{1}=\{1 /(a+c>1), 1 /(b+d>1)\}, \theta_{2}=\{1 /(a+c>1), 0 /(b+d>1)\}, \\
& \theta_{3}=\{0 /(a+c>1), 1 /(b+d>1)\}, \theta_{4}=\{0 /(a+c>1), 0 /(b+d>1)\} .
\end{aligned}
$$

Then we have

$$
\begin{aligned}
& \theta_{1}{ }^{\#}=(a+c>1) \wedge(b+d>1), \theta_{2}{ }^{\#}=(a+c>1) \wedge(b+d \leq 1), \\
& \theta_{3}{ }^{\#}=(a+c \leq 1) \wedge(b+d>1), \theta_{2}{ }^{\#}=(a+c \leq 1) \wedge(b+d \leq 1) .
\end{aligned}
$$

For the condition

$$
\mathrm{C}=(a+c>1 \wedge y \geq 1-c) \vee(b+d>1 \wedge y \geq b)
$$

we have

$$
\begin{aligned}
& \mathrm{C} \theta_{1}=((y \geq 1-c) \vee(y \geq b)), \mathrm{C} \theta_{2}=(y \geq 1-c), \\
& \mathrm{C} \theta_{3}=(y \geq b), \mathrm{C} \theta_{4}=0 .
\end{aligned}
$$

Hence,

$$
\mathrm{r}\left(\mathrm{C} \theta_{1}\right)=\{1-c, b\}, \mathrm{r}\left(\mathrm{C}_{2}\right)=\{1-c\}, \mathrm{r}\left(\mathrm{C}_{3}\right)=\{b\}, \mathrm{r}\left(\mathrm{C}_{4}\right)=\{\} .
$$

Using the formula presented in the second row of Table 3 we obtain the expression $g$ coinciding with what is presented in Example 2.

\section{References}

1. Straccia U. Reasoning and Experimenting within Zadeh's Fuzzy propositional Logic. Technical Report 2000-b4-011. Instituto di Elaborazione dell'Informazione, Consiglio Nazionale delle Richerche, Pisa, Italy, 2000.

2. Hahnle R. Automated Deduction in Multi-Valued Logic. International Series of Monographs on Computer Science 10. Oxford University Press, 1995. 